\title{
Supernova Neutrinos search with the LVD experiment: the 2019 update
}

\section{Vigorito* on behalf of the LVD Collaboration}

Università \& INFN Torino, Italy

E-mail: vigoritodto.infn.it

\section{G. Bruno}

INFN Laboratori Nazionali del Gran Sasso, Italy

E-mail: Gianmarco.brunodelngs.infn.it

\section{W. Fulgione}

INFN Laboratori Nazionali del Gran Sasso, Italy

E-mail: fulgioneeto.infn.it

\section{A. Molinario}

INFN Laboratori Nazionali del Gran Sasso, Italy

E-mail: andrea.molinariodengs.infn.it

The Large Volume Detector (LVD) at the INFN Gran Sasso National Laboratory, Italy, is a neutrino observatory designed to study low energy neutrinos from gravitational stellar collapses. The detector, 1 kton of liquid scintillator, is sensitive to core-collapse supernovae via neutrino burst detection with $100 \%$ efficiency over the Galaxy. In this paper we summarize the results of the last run, lasting from 2014, January $1^{\text {st }}$ to 2019 , June $6^{\text {th }}$ for a total live time of 1978 days. In the lack of a positive observation in this data set and including all previously published results since 1992 for a total of 9313 days, the upper limit on the rate of core collapse and failed supernova explosions out to distances of $25 \mathrm{kpc}$ is 0.09 year $^{-1}$ at $90 \%$ c.l..

36th International Cosmic Ray Conference -ICRC2019-

July 24th - August 1st, 2019

Madison, WI, U.S.A.

\footnotetext{
* Speaker.
} 


\section{Introduction}

Gravitational Stellar Collapses (GSC) are astrophysical events of great interest. Because of the complexity of the problem, the modelling of the physical processes is still in evolution, but it is in general accepted that the role of neutrinos is critical to allow the supernova to form out of a collapse [四].

The detection of the neutrino signal from the SN 1987A marked the beginning of a new era in neutrino astrophysics (e.g. in [Q, [], 田]) and, in spite of some unresolved controversies [[]], opened the way to the Neutrino Astronomy.

At the time of next event in our Galaxy the correlated neutrino emission will be eventually detected by different detectors. Such rare event is expected to happen in the Galaxy every 30-50 years [6] and therefore enforces to set up detectors which last for decades with a very high duty cycle.

Actually, since light can be partially or totally absorbed by dust in the Galactic plane while neutrinos are not (see the recent discussion by [ [ $]$ ]), large long-term neutrino detectors are the most suited ones to observe the Galaxy and search for core collapse supernovae ( $\mathrm{ccSN})$. Neutrino detectors are also sensitive to collapsing objects that fails to explode becoming black holes, the so-called failed supernovae (fSN). Those are expected to emit a neutrino signal even stronger[[]], although shorter in time than ccSN.

\section{The LVD detector}

The Large Volume Detector (LVD) is located underground at a depth of $1400 \mathrm{~m}$ under rock (minimal depth $3000 \mathrm{~m}$ w.e.), in the INFN Gran Sasso National Laboratory (Italy). The experiment consists of an array of 840 scintillator counters, $1.5 \mathrm{~m}^{3}$ each, viewed from the top by three photomultipliers (PMTs) and arranged in a modular geometry[Q]. This modularity allows LVD to achieve a very high duty cycle, that is essential in the search of unpredictable sporadic events. Failures involving one or more counters do not affect, in general, other counters. The detector maintenance can be done during data acquisition by stopping only the part that needs to be maintained, even a single counter. This peculiarity allows a dynamic active mass $\mathrm{M}_{a c t}$ and a high duty cycle. The experiment has been in operation since 1992, June $9^{\text {th }}$ after a short commissioning phase, its mass increasing from $300 \mathrm{t}$ to its final one, $1000 \mathrm{t}$, at time of building phase completion in January 2001. Duty cycle and active mass along the experiment life, up to 2019, June $6^{\text {th }}$ are shown in Fig. W.

\subsection{Neutrino interaction channels in LVD}

Neutrinos can be detected in LVD through charged current (CC) and neutral current (NC) interactions on proton, carbon nuclei and electrons of the liquid scintillator but also on the iron nuclei of the support structure $(850 \mathrm{t})$. In the latter case the products of interactions in iron can eventually reach the scintillator and be detected [ए]]. The total target thus consists of $8.3 \cdot 10^{31}$ free protons, $4.3 \cdot 10^{31} \mathrm{C}$ nuclei, $3.4 \cdot 10^{32}$ electrons in the scintillator and of $9.7 \cdot 10^{30} \mathrm{Fe}$ nuclei. The main neutrino interaction in LVD in case of a $v$ burst from GSC is the Inverse Beta Decay (IBD), as it can be seen in Table $\mathbb{W}$, where all other relevant neutrino interaction channels are also shown. Given the relevance of the IBD, the LVD trigger has been optimized for the detection of both 

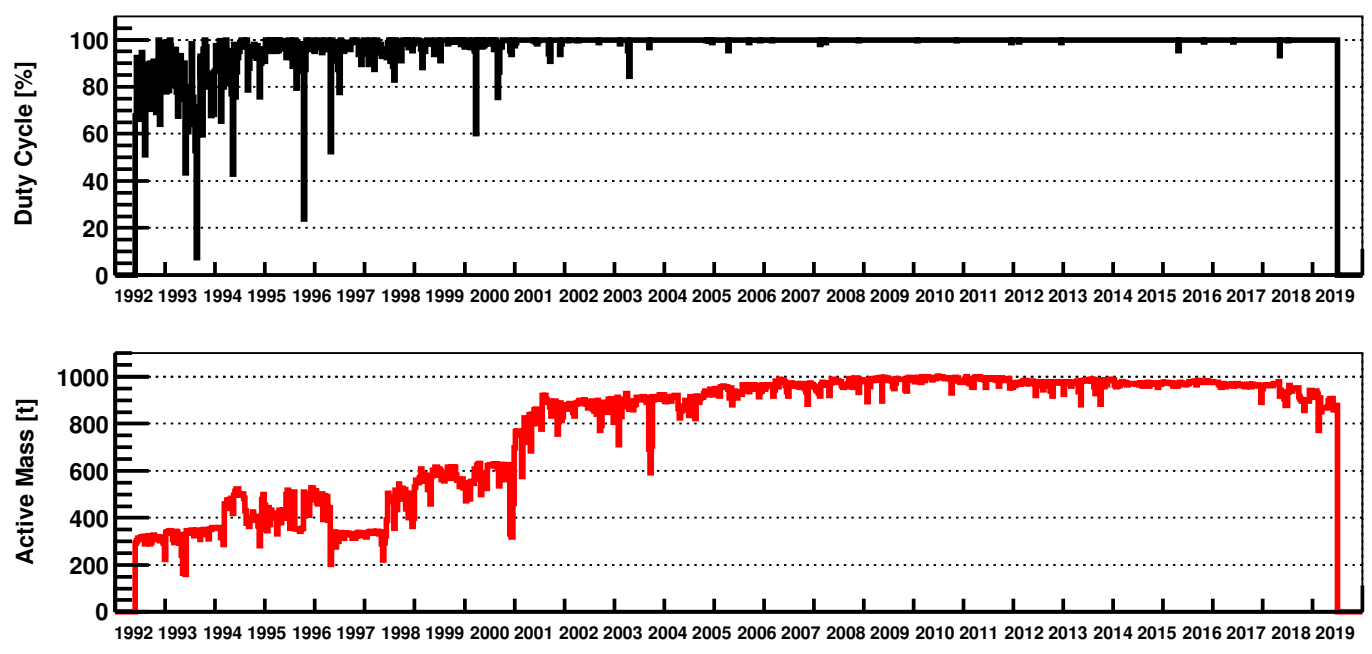

Figure 1: LVD duty cycle and active mass as a function of time from 1992, June $9^{\text {th }} 1992$ to 2019, June $6^{\text {th }}$.

products of this interaction, namely the positron and the neutron. Each PMT is thus discriminated at two different threshold levels, the higher one $\left(\mathscr{E}_{H} \simeq 4 \mathrm{MeV}\right.$ in the present settings) is also the main trigger condition for the detector array. The lower one $\left(\mathscr{E}_{L} \simeq 0.5 \mathrm{MeV}\right)$ is in turn active only in a $1 \mathrm{~ms}$ time-window following the trigger, allowing the detection of $(n, p)$ captures, the marker of a possible IBD interaction in the detector. Once a trigger is identified, the charge and time of the three summed PMTs signals are stored in a memory buffer. One millisecond after the trigger, all memory buffers are read out.

\begin{tabular}{cccc}
\hline \hline & $v$ interaction channel & $\mathrm{E}_{v}$ threshold & $\%$ \\
\hline 1 & $\bar{v}_{\mathrm{e}}+\mathrm{p} \rightarrow \mathrm{e}^{+}+\mathrm{n}$ & $(1.8 \mathrm{MeV})$ & $(88 \%)$ \\
2 & $v_{\mathrm{e}}+{ }^{12} \mathrm{C} \rightarrow{ }^{12} \mathrm{~N}+e^{-}$ & $(17.3 \mathrm{MeV})$ & $(1.5 \%)$ \\
3 & $\bar{v}_{\mathrm{e}}+{ }^{12} \mathrm{C} \rightarrow{ }^{12} \mathrm{~B}+e^{+}$ & $(14.4 \mathrm{MeV})$ & $(1.0 \%)$ \\
4 & $v_{\mathrm{i}}+{ }^{12} \mathrm{C} \rightarrow v_{\mathrm{i}}+{ }^{12} \mathrm{C}^{*}+\gamma$ & $(15.1 \mathrm{MeV})$ & $(2.0 \%)$ \\
5 & $v_{\mathrm{i}}+e^{-} \rightarrow v_{\mathrm{i}}+e^{-}$ & $(-)$ & $(3.0 \%)$ \\
6 & $v_{\mathrm{e}}+{ }^{56} \mathrm{Fe} \rightarrow{ }^{56} \mathrm{Co}^{*}+e^{-}$ & $(10 . \mathrm{MeV})$ & $(3.0 \%)$ \\
7 & $\bar{v}_{\mathrm{e}}+{ }^{56} \mathrm{Fe} \rightarrow{ }^{56} \mathrm{Mn}+e^{+}$ & $(12.5 \mathrm{MeV})$ & $(0.5 \%)$ \\
8 & $v_{\mathrm{i}}+{ }^{56} \mathrm{Fe} \rightarrow v_{\mathrm{i}}+{ }^{56} \mathrm{Fe}^{*}+\gamma$ & $(15 . \mathrm{MeV})$ & $(2.0 \%)$ \\
\hline
\end{tabular}

Table 1: The $v$ interaction channels detectable in LVD. The last column shows the relative fractions of events for any interaction channel in case of detection of a $v$ burst from GSC. The parametric emission model [ए]] is assumed.

The number of detected events in LVD for a neutrino burst from ccSN have been evaluated via a parameterized model based on a maximum likelihood procedure on the data from SN 1987A [U]]. The resulting average $\bar{v}_{e}$ energy is $\bar{E}_{\bar{v}_{e}}=14 \mathrm{MeV}$, being $E_{b}=2.4 \cdot 10^{53}$ erg the total radiated energy. Energy equipartition and normal mass hierarchy for neutrino oscillations are also assumed. At a reference distance $D=10 \mathrm{kpc}$ for the ccSN, we found that a total of 300 events (260 at $E_{v} \geq 10$ 
$\mathrm{MeV}$ ) are expected in LVD with a $1 \mathrm{kt}$ active mass, $88 \%$ of which are due to IBD (see details in [ए2]).

By a similar procedure we also evaluated the number of neutrino signals in LVD in the case of fSN. A bunch of 500 events per kton at $E_{v} \geq 10 \mathrm{MeV}$ in $0.36 \mathrm{~s}$ is expected at $10 \mathrm{kpc}$ in the worst theoretical model.

\section{Search for neutrino bursts}

LVD science runs started in June 1992 with increasing mass configurations, being $300 \mathrm{t}$ the minimal mass that allows LVD to be fully sensitive to neutrino bursts from GSC up to distance $D<25 \mathrm{kpc}$. The results of the search for neutrino bursts with LVD have been periodically reported

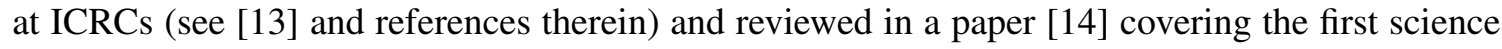
run including data taking of the years since 1992 up to 2013. Here we focus on the results of the analysis of the last run, since 2014, January $1^{\text {st }}$ to 2019 , June $6^{\text {th }}$, for a total live-time of 1978 days, corresponding to an effective duty cycle of $99.8 \%$. The data set includes $1.86 \cdot 10^{9}$ triggers reduced to 4097531 in the [10-100] $\mathrm{MeV}$ energy range after muon rejection and quality cuts. The time evolution of these rates are shown in figure $\square$. Table $\square$ summarizes the features of the last run: average trigger rates before and after selections, active mass, total exposure and livetime.

\begin{tabular}{cccccccc}
\hline \hline & $R_{\text {tot }}$ & $R_{10}(E \geq 10 \mathrm{MeV})$ & $R_{L}(E \geq 0.5 \mathrm{MeV})$ & $\bar{M}_{\text {act }}$ & Exposure & live time & live time $(M \geq 300 \mathrm{t})$ \\
& {$\left[s^{-1} \cdot t^{-1} \cdot 10^{-4}\right]$} & {$\left[s^{-1} \cdot t^{-1} \cdot 10^{-4}\right]$} & {$\left[s^{-1} \cdot t^{-1}\right]$} & {$[\mathrm{t}]$} & {$[t \cdot$ year $]$} & {$[$ days $]$} & {$[$ days $]$} \\
\hline Last run & 111 & 0.26 & 262 & 928 & 5030 & 1978 & 1978 \\
\hline
\end{tabular}

Table 2: Data set features for the last run period.

After the quality cuts the total counting rate $f$ results to be quite stable (bottom thick line in figure 2). The distribution of time delay between triggers is shown in figure B, normalized to a reference background rate $\left(\delta t_{\text {norm }}=\delta t \cdot f / f_{\text {ref }}\right.$, being $f_{\text {ref }}=0.03 \mathrm{~s}^{-1}$ the typical average rate for the full LVD detector in the [10-100] MeV range) to take into account the variable active mass configurations, as shown in figure $\mathbb{m}$. The LVD events behave as a stochastic time series well described by the Poisson statistics, as shown by the exponential fit in figure [B.

\subsection{The selection algorithm}

To search for supernova neutrino bursts, we analize the time series of the selected events and look for clusters. While to provide the SNEWS, the on-line network of running neutrino detectors [ㄷ]], with a prompt alert we use in the burst search method (on-line mode) a fixed time window (20s) [16], in this analysis (off-line mode) we consider different burst durations up to $100 \mathrm{~s}$ as discussed in detail in [ㄱ] . In both cases the selection is essentially a two-step process.

In the first step, we analyze the entire time series to search for clusters of events. The rationale of the search is that every $n$-th event could be the first of a possible neutrino burst. As we do not know a priori the duration of the burst, we consider all clusters formed by the $n$-th event and its successive ones. Namely, the $n$-th and the $(n+1)$-th ones define a cluster of multiplicity $m=2$; the $n$-th,$(n+1)$-th, $(n+2)$-th ones define another cluster of $m=3$, and so on. The duration of each 


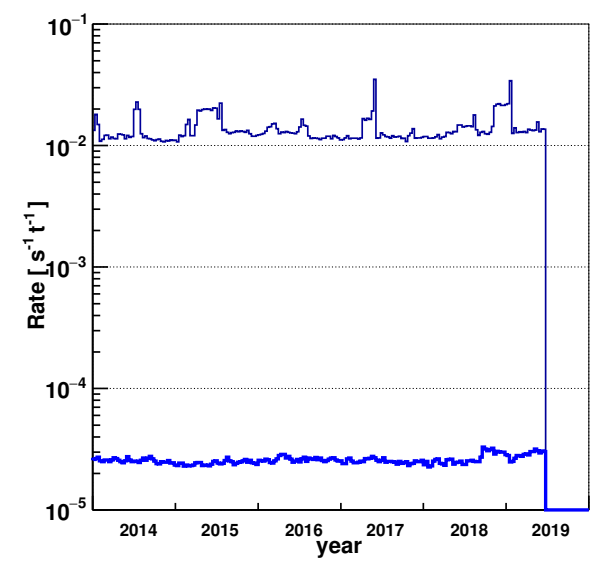

Figure 2: LVD counting rate as a function of time in the last run: top (thin) and bottom (thick) lines show the total rate pre and post quality and energy selection cuts respectively.

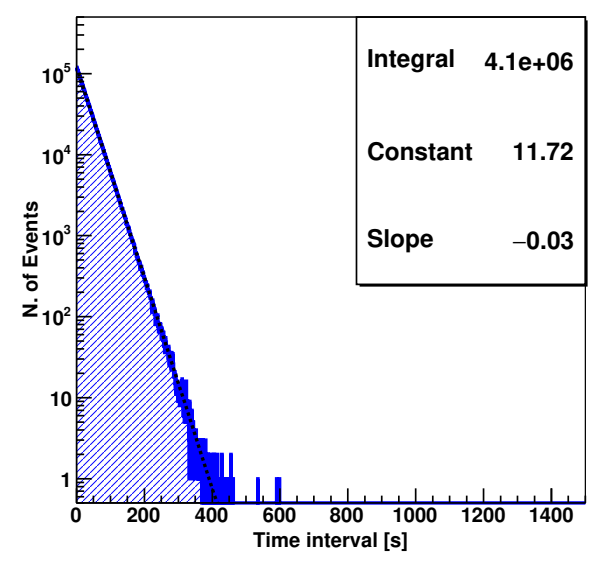

Figure 3: Distribution of normalized time delay between triggers of the last run. The exponential fit shows that the stochastic time serie is well in agreement with the expected Poisson statistics.

cluster is given by the time difference $\Delta \mathrm{t}$ between the first event $n$-th and the last one of each sequence up to a maximum value of $\Delta \mathrm{t}_{\max }=100 \mathrm{~s}$. The analysis is then applied to the $(n+1)$ th event, and iteratively to all LVD events. The advantage of the described analysis is that it is unbiassed with respect to the duration of the possible neutrino burst, unknown a priori. The choice of $\Delta \mathrm{t}_{\max }=100 \mathrm{~s}$ is very conservative as it well exceeds the expected duration of a neutrino burst from ccSN (tens of seconds) and even more from fSN (ms-s time scale).

The second step of the process consists in determining if one or more among the detected clusters are neutrino bursts candidates. To this aim, we associate to each of them (characterized by multiplicity $\mathrm{m}_{\mathrm{i}}$ and duration $\Delta t_{i}$ ) the imitation frequency $\mathrm{F}_{\mathrm{im}_{\mathrm{i}}}$. This quantity represents the frequency with which background fluctuations can produce, by chance, clusters with multiplicity $m \geq m_{i}$ and duration $\Delta t_{i}$. As shown in [ए]], it depends on $\left(\mathrm{m}_{\mathrm{i}}, \Delta \mathrm{t}_{\mathrm{i}}\right)$, on the instantaneous background frequency, $\mathrm{f}_{\mathrm{bk}_{\mathrm{i}}}$ and on the maximum cluster duration chosen for the analysis, $\Delta \mathrm{t}_{\max }$, and can be written as:

$$
\mathrm{F}_{\mathrm{im}}=\mathrm{f}_{\mathrm{bk}}^{2} \Delta \mathrm{t}_{\max } \sum_{\mathrm{k} \geq \mathrm{m}_{\mathrm{i}}-2} \mathrm{P}\left(\mathrm{k}, \mathrm{f}_{\mathrm{bk}_{\mathrm{i}}} \Delta \mathrm{t}_{\mathrm{i}}\right)
$$

where $\mathrm{P}\left(\mathrm{k}, \mathrm{f}_{\mathrm{bk}_{\mathrm{i}}} \Delta \mathrm{t}_{\mathrm{i}}\right)$ is the Poisson probability to have $\mathrm{k}$ events in the time window $\Delta \mathrm{t}_{\mathrm{i}}$ if $\mathrm{f}_{\mathrm{bk}}$ is the background frequency.

Given the long duration of the LVD data set, we choose $1 / 100$ year $^{-1}$ as imitation-frequency threshold, $F_{\mathrm{im}}^{\mathrm{th}}$. This means that a cluster $\left(\mathrm{m}_{\mathrm{i}}, \Delta \mathrm{t}_{\mathrm{i}}\right)$ is considered a neutrino burst candidate if:

$$
\sum_{\mathrm{k} \geq \mathrm{m}_{\mathrm{i}}-2} \mathrm{P}\left(\mathrm{k}, \mathrm{f}_{\mathrm{bk}_{\mathrm{i}}} \Delta \mathrm{t}_{\mathrm{i}}\right)<\frac{\mathrm{F}_{\mathrm{im}}^{\mathrm{th}}}{\mathrm{f}_{\mathrm{bk}}^{2} \cdot \Delta \mathrm{t}_{\max }} .
$$

The introduction of the imitation frequency has a double advantage. From the viewpoint of the search for neutrino bursts, it allows us to define a priori the statistical significance of each cluster in terms of the background frequency, in general not constant but actually changing with the detector 
active mass. Also, it allows us to monitor the performance of the search algorithm and the stability of the detector by changing the imitation frequency threshold. Namely, we study the distributions of time differences between consecutive clusters for three different values of imitation frequency $\left(\mathrm{F}_{\mathrm{im}}<1\right.$ day $^{-1}$, week ${ }^{-1}$, month $\left.^{-1}\right)$. Given the limited statistics we get when considering only the current run $\left(278,41\right.$ and 12 clusters detected at $\mathrm{F}_{\mathrm{im}}<1$ day $^{-1}$, week ${ }^{-1}$, month ${ }^{-1}$ respectively), we perform this study including the data already published for the period 1992-2013 [14]]. This results in a total of 1401, 206, 57 clusters for the 3 imitation frequencies, respectively. The distributions of the time differences between consecutive clusters are shown in figure $\mathrm{t}$ for $\mathrm{F}_{\mathrm{im}}<1$ day $^{-1}$ (black solid line), $\mathrm{F}_{\mathrm{im}}<1$ week $^{-1}$ (green solid line) and $\mathrm{F}_{\mathrm{im}}<1$ month $^{-1}$ (blue solid line). The superimposed dotted lines are pure exponential fits to each distribution following the expected Poissonian behaviour. This result shows that the search algorithm and the detector are quite under control over the whole period of data taking.

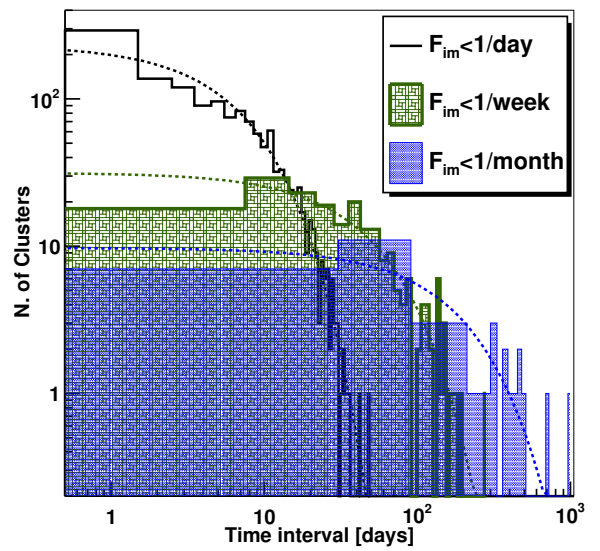

Figure 4: Distribution of time intervals beween consecutive clusters (solid lines) fitted by Poisson laws (dashed lines). $\quad$ All data since 1992 have been included here.

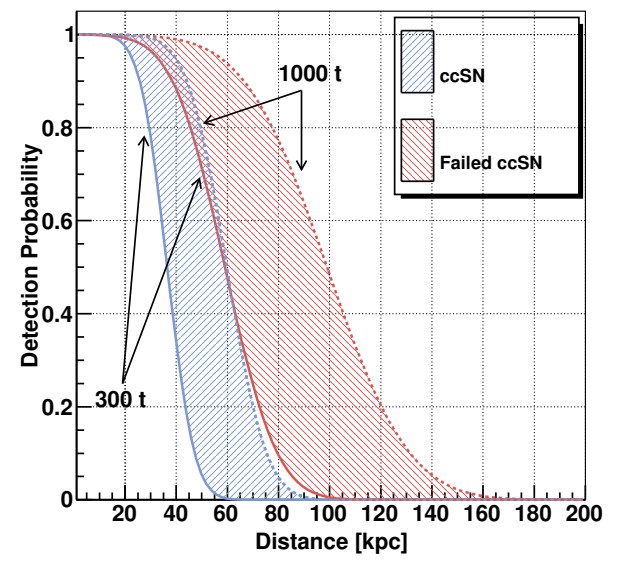

Figure 5: LVD detection probability versus source distance for the $F_{\text {im }}=1 / 100$ year $^{-1}$ for both ccSN and $\mathrm{fSN}$ in the range of minimum $(300 \mathrm{t})$ and maximum $(1000 \mathrm{t})$ allowed active mass.

Finally, the detection probability associated to the off-line method is shown in Figure $\square$ for the chosen imitation frequency of $1 / 100$ year $^{-1}$ as a function of the distance of the collapse. The blue band corresponds to the case of standard ccSN: the solid (dashed) line represents an active mass of 300 (1000) t. The red band evaluates the detection probability in the case of stellar collapses ending into black-holes by using a similar procedure as above.

According to that we can conclude that LVD is fully efficient to ccSN or fSN within a radius of 25 kpc provided the detector active mass is greater than $300 \mathrm{t}$.

\section{Results}

By analyzing the cleaned time serie of 4097531 events of the last run, selected as described in Section 13 and collected over 1978 days of data-taking, we studied 9877087 clusters with multiplicity $m \geq 2$ and $\Delta \mathrm{t} \leq 100 \mathrm{~s}$. 


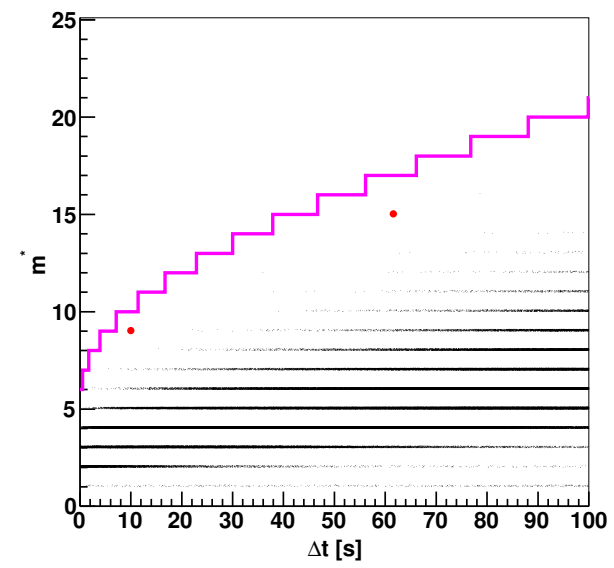

Figure 6: Distribution of detected clusters in the space $\left(\Delta t, m^{*}\right)$. Red dots represent clusters with imitation frequency less than $\mathrm{F}_{\mathrm{im}}=1$ year $^{-1}$. The purple line corresponds to $\mathrm{F}_{\mathrm{im}}^{\text {th }}=1 / 100$ years $^{-1}$.

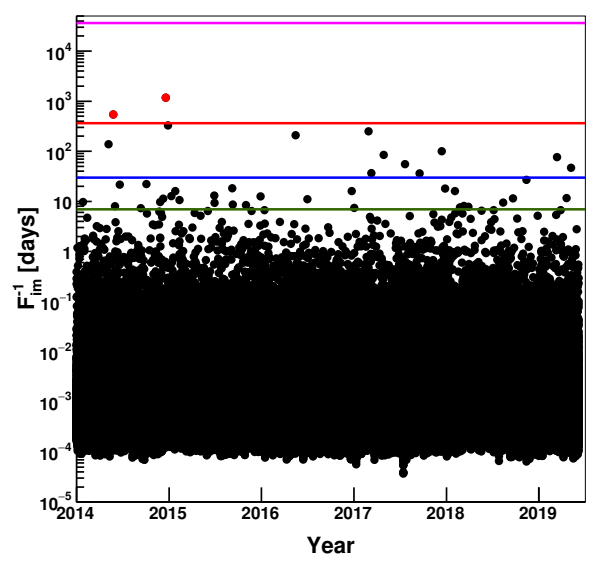

Figure 7: Distributions of detected clusters versus time for the present data set. Red dots represent clusters with imitation frequency less than $F_{i m}=1$ year $^{-1}$. Green, blue, red and purple lines corresponds to $\mathrm{F}_{\mathrm{im}}^{\text {th }}=1$ week $^{-1}, \mathrm{~F}_{\mathrm{im}}^{\text {th }}=1$ month $^{-1}$, $\mathrm{F}_{\mathrm{im}}^{\text {th }}=1$ year $^{-1}, \quad \mathrm{~F}_{\mathrm{im}}^{\text {th }}=1 / 100$ year $^{-1}$, respectively.

Figure 6 shows the correlation between the cluster duration, $\Delta \mathrm{t}$, and the normalized multiplicity, $m^{*}$, which takes into account the different instantaneous background rate at time of detection and allows to put all clusters toghether normalized to the reference background rate, $f_{\text {ref }}$. Details on the normalization procedure are discussed in [14]. Figure $\square$ shows the absolute imitation frequency of all clusters (i.e. their significance) as a function of time. It is evident that the occurrence of clusters with different $F_{\text {im }}$ over 1978 days of measurement is quite uniform. In both figures the purple line represents the expectations for a $F_{\text {im }}^{\text {th }}$ of $1 / 100$ year $^{-1}$, i.e., the threshold to define a cluster as a neutrino-burst candidate with LVD. Two clusters have a $F_{i m}<1$ year $^{-1}$, being the maximum detected significance $\left(\mathrm{F}_{\mathrm{im}}\right)^{-1}=3.2$ year, associated to a cluster of 14 events lasting about $61 \mathrm{~s}$. Actually both clusters have been checked in terms of energy spectra and low energy signals that may be the signature of the IBD interactions. They are fully compatible with chance coincidence among background signals. We conclude that no evidence is found for ccSN or fSN during the considered data-taking period. Taking into account the total live-time of LVD (9313 days) the

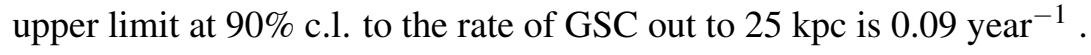

\section{Conclusions}

In this paper we have summarized the results of the search for neutrino bursts from GSC performed with LVD data taken over the last run since 2014, January $1^{\text {st }}$ to 2019 , June $6^{\text {th }}$ with the off-line method. Neutrino burst candidates are selected as clusters of events, with duration up to $100 \mathrm{~s}$, statistically selected by having an imitation frequency less than $1 / 100$ year $^{-1}$. This makes our search model-independent, as the duration of a neutrino burst due to a supernova explosion is 
unknown. The knowledge of the background as well as its long-term stability are of essence for evaluating the probability of each found cluster. Out of the $\sim 9.9$ millions of detected clusters, we have found that none has an imitation frequency less than $1 / 100$ year $^{-1}$ in the current run. We have thus concluded that no evidence has been found for GSC occurred up to $25 \mathrm{kpc}$ during the period of observation. Taking into account all previous data since 1992 for a total livetime of 9313 days, we have set an upper limit of 0.09 year $^{-1}$ at $90 \%$ c.l., this being the most stringent limit ever achieved by the observation of supernovae through neutrinos in the entire Galaxy.

\section{References}

[1] Bethe H.A. \& Wilson J.R. 1985, ApJ., 295, 14.

[2] Hirata, K et al. 1987, PhRvL, 58, 1490.

[3] Bionta R.M. et al. 1987, PhRvL, 58, 1494.

[4] Alekseev E.N. et al. 1987, JTPL, 45, 589.

[5] Aglietta M. et al. 1987, EL, 3, 1315.

[6] R.Diehl et al. 2006, Nature, 439, 45.

[7] Adams Scott M. et al., 2013, ApJ, 778:164.

[8] Nazakato k., Sumiyoshi K., Suzuki H. and Yamada S., 2008, PhRvD, 78, 083014.

[9] Aglietta, M. et al., 1992, NCimA, 105, 1793.

[10] Agafonova N.Yu. et al., 2007, APh, 27, 254-270.

[11] Pagliaroli G. et al. 2009, APh, 31, 163.

[12] Molinario A. 2012 , Ph.D. Thesis, Dep. of Physics, University of Torino.

[13] Vigorito C., Bruno G., Fulgione W. and Molinario A., 2017, Proc. of $35^{\text {th }}$ ICRC (Busan, Korea).

[14] Agafonova N.Yu. et al., 2015, ApJ, 802, 47.

[15] Antonioli P. et al., 2004, NJPh, 6, 114.

[16] Agafonova N.Yu. et al., 2008, APh, 28, 516.

[17] Fulgione W., Mengotti-Silva N. \& Panaro L., 1996, NIMPA, 368, 512. 